\title{
Ethical, legal and clinical aspects of live surgery in urology - contemporary issues and a glimpse of the future
}

\author{
Alin Adrian Cumpanas ${ }^{1}$, Ovidiu Catalin Ferician ${ }^{1}$, Silviu Constantin Latcu ${ }^{1}$, Catalin Pricop ${ }^{2}$, Razvan Tiberiu Bardan ${ }^{1}$ \\ ${ }^{1}$ Department of Urology, Victor Babes University of Medicine and Pharmacy, Timisoara, Romania \\ ${ }^{2}$ Department of Urology, Gr.T. Popa University of Medicine and Pharmacy, lasi, Romania
}

Videosurgery Miniinv 2017; 12 (1): 1-6 DOI: https://doi.org/10.5114/wiitm.2017.66502

\begin{abstract}
Beside dry and wet lab training, simulators, video tapes, fellowships and clinical visits, live surgery has gained popularity during the last years, being an attraction point at large scientific meetings and at postgraduate courses as well. This type of surgical training raises both ethical and legal issues. Thus, there are professional societies that have banned such meetings, mainly due to safety reasons for the patient. The current article aims to identify and to discuss ethical and legal issues related to the topic, advantages, disadvantages and weak points of this emerging challenge for modern medicine, trying to analyze the issues from all relevant points of view: those of the patient, the surgeon and the session attendant.
\end{abstract}

Key words: live surgery, urology, surgical training.

\section{Introduction}

The tremendous development of surgical devices and, by consequence, of surgical techniques within the last decades urges urologists to continue to adapt their knowledge and skills to the newest technologies and techniques. Continuous medical education (CME) for the surgeons means both theoretical and practical up-dates, including learning the newest techniques or using the new devices. For instance, within the last decade bipolar transurethral resection of the prostate (TURP), photoselective vaporization of the prostate, thulium/ holmium laser enucleation of the prostate, flexible ureteroscopy, mini percutaneous nephrolithotomy (mini PCNL) and micro PCNL, laser stone disintegration, high intensity focus ultrasound (HIFU), retrograde intrarenal surgery (RIRS), endoscopic combined intrarenal surgery (ECIRS), fusion prostate biopsy, single port laparoscopy, natural orifice translumenal endoscopic surgery, 3D laparoscopy, robotic surgery and many others were introduced into routine clinical practice.

In these conditions, the urologist (and the surgeon, in general) must learn as much as possible within a short period of time. Otherwise, they will soon become overwhelmed by the amount of knowledge they will have to gain.

How can the surgical procedures and specific surgical steps be learnt? The classic ways include dry lab practice, wet lab practice, simulators, observation in the operation room, assisting the surgeon, performing surgical procedures under direct supervision of a tutor, textbooks, journals, recorded surgical procedures on DVDs or on the internet. However, it is obvious that the above-mentioned ways of training can ensure only the "basics", the core surgical training, as the newest technologies and techniques develop very rapidly. That is the main

\section{Address for correspondence}

Cumpanas Alin Adrian MD, PhD, FEBU, Department of Urology, Victor Babes University of Medicine and Pharmacy, Bdul L. Rebreanu 156, 300723 Timisoara, Romania, phone: +40 723 299937, e-mail: alincumpanas@hotmail.com 
reason why there is a continuous pressure from the attendees at scientific meetings to have live surgery sessions.

Within the last years, the major European urological meeting - the Annual Congress of the European Association of Urology - gained more than 10000 attendants every year. During a dedicated full-day live-surgery session, most of the time, the main auditorium was full, more than 4000 urologists attending the session. A similar situation is encountered in live surgery sessions of the American Urological Association (AUA) Annual Meetings as well as at the postgraduate courses where live surgery sessions are scheduled. Thus, a tremendous interest for live surgery does exist.

On the other hand, in 2006, the Japanese Society of Thoracic Surgeons stopped live surgery sessions because a patient died after the repair of an aortic aneurysm. Thereafter, most other Japanese societies, including the Japanese Urology Association, did the same. Thereafter, the American College of Surgeons and the American College of Obstetricians and Gynecologists banned live surgery sessions [1]. The American Association of Thoracic Surgery and the Society of Thoracic Surgery were the pioneers in rising questions regarding the benefits and risks of live surgery sessions and for establishing guidelines for organizing this type of educational events instead of banning them [2]. The European Association of Urology (EAU) established a set of rules for organizing live sessions as well [3].

However, beside these actions, trying to establish rules in this new trend of modern medicine, ethical issues still remains to be discussed, as - apart from the interest in having highly successful meetings the subject has to be evaluated at least from three points of view: the patient's (the benefits and the risks he/she is exposed to), the surgeon's (who performs the procedure) and the attendant doctor/live surgery session participant (the advantages and disadvantages of this type of medical education versus other types of education).

\section{The patient's perspective - beyond the "therapeutic misconception"}

The three most important issues from the patient's point of view are privacy, informed consent and the safety of the procedure. Beside the patient's confidence in the physician, the above-mentioned elements form the basis of every patient-physician relationship. Are these issues always granted during a live surgery session? It is ethical for the patient to be exposed to a live surgery session? Does live surgery offer to the patient the best chances to be treated with the best results for a certain disease? If yes, in which conditions? If not, what are the weak points and risks, and how they can be corrected?

First of all, the privacy of the patient is a right which should never be infringed. The privacy of the patient should be always guaranteed during such meetings, taking into consideration that his/her medical history and investigation results would be presented to the auditorium before the procedure. Moreover, care must be taken in order not to allow the transmission from the theatre to the auditorium until the patient has not been draped. If positioning of the patient is important for the surgery (being a new approach, etc.), the face of the patient must be covered or the image be blurred.

The informed consent for the procedure is mandatory for all types of surgical procedures both from legal and ethical points of view. Due to the special relation between the patient and the doctor, which can be perceived as a paternalistic one (this aspect varying from country to country, from culture to culture), a power gradient in favor of the doctor usually exists [4], and care must be taken when this aspect adds to the pressure of being a subject of a live-surgery event. The "therapeutic misconception" - the patient's temptation to deny the possibility of having disadvantages by participating in a certain form of treatment [5] - or the therapeutic misestimating and unrealistic optimism should be discussed with the patient, in order to obtain valid informed consent [6].

In the case of a live-surgery event, beside the explanation of the procedure itself and the alternatives to this treatment, it should include the explanation of how the live session will be done, who will be in the surgical team, their experience, and the size of the audience (as, sometimes, it might influence the patient's decision to participate in the event due to the high potential to breach confidentiality) [4, 7]. Care must be taken to avoid situations where undue pressure might be placed on the patient facing the possibility of having a renowned surgeon performing his operation [7]. For this reason, a detailed explanation of an alternative to live surgery, as well as the risks and benefits, should be presented to 
the patient. Choosing a patient for a certain procedure should be clearly in the benefit of the patient, bearing in mind the cost-effectiveness ratio (otherwise, if the procedure - an expensive one - is done instead of a simpler and cheaper one, we face unjustified spending of resources which is, also, an ethical issue).

It is advisable that the potential added risk due to surgeon distraction during live surgery be explained to the patient. Another aspect which should be taken into consideration is how the written consent was obtained (volunteer vs. paid). There are countries where the patient is paid to sign the consent form or they might do this to be waived from paying the costs of the procedure or because they are not offered an alternative to their disease. In every situation of this type, it is advisable that the surgeon refrains from performing surgery $[4,7]$.

The case selection and preparation for certain procedures implies longer waiting times than usual. Although it is very hard to estimate due to many bias factors, there are data from the literature estimating an average waiting time of 9 days longer than usual [8]. In urology, for the majority of surgical procedures this is not an excessive period except for testicular cancer or for bladder cancer procedures where every day is important as the regular time from diagnosis to treatment takes a few weeks [3]. However, the patient could have an unnecessary/harmful delay in order to be scheduled for the event or, on the other hand, if the appropriate case is not available, a patient who would not benefit $100 \%$ from the scheduled procedure could be considered for the live surgery [9].

For all the above reasons, a patient advocate (a person not involved in the live-surgery session) should be offered to the patient as a third-party counselor and advisor who aims that the patient's rights are respected.

\section{The surgeon's perspective - "first do no harm" and beyond}

The Hippocratic oak requires that doctors "first do no harm", and this should be the very first ethical issue to be taken into consideration by every surgeon invited to perform such surgery. Is the live surgery event necessary for the peers? Can the best results be obtained in the particular situation of live surgery? Is the patient exposed to additional risks?
How to surpass the ethical, legal and technical problems raised by such events?

The live surgery sessions are not easy tasks for surgeons performing them. The first aspect is related to the itinerant surgeon, who often arrives with jet-lag or sleep deprived $[1,9]$. That is why the surgeon should carefully schedule his travel dates according to the surgery he has to perform.

From the very beginning it should mentioned that the surgeon must be competent in all that involves the surgical procedure(s) which will be done. This includes the technique, the management of complications and alternative techniques which could be done if the foreseen procedure is not possible [7]. The European Association of Urology Live Surgery Endorsed Guide requires that the local hospital nominates a director of the live surgery event who should review the guest's credentials and CV, ensures that the guest surgeon holds operating privileges in his/her country and is granted to operate in the host hospital.

On the other hand, the surgeon's role should be to disseminate knowledge, and "live surgery should never become a circus for showmen with the intention to promote the institution or the ego: We should stay away from this at all times!" [10].

It is important that any financial disclosure (by the host institution, by a guest or by the patient) should be made before the event. Moreover, the guest surgeon should be named in the patient's informed consent and there should be clarification of the legal liabilities of both the surgeon and the facility where the live surgery will be performed $[3,7]$.

It is recommended that the surgeon visit and examine the patient and observe the investigations, especially the CT, X-ray reports, etc. Otherwise, there is a risk of pitfalls or to be "educated by humiliation" as Professor Mundy describes his experience as a guest surgeon [11].

Care must be taken to visit the operating room before the surgery, to check the instruments, equipments and devices, to discuss with the assistants, to establish the operating team, to discuss the specific tasks. Sometimes, the surgeon can prefer as an assistant one of his fellows (who is familiar with a certain procedure) and can even bring the instruments he/she is familiar with [9]. An alternative to this is broadcasting live surgery from the surgeon's own institution, especially for the most complex procedures which need special conditions intraoperatively 
and postoperatively or very expensive equipment (e.g. robotic surgery). This would prevent distress situations created by different equipment/instrument malfunction or by the team function itself, due to differences in habits and behaviors. There are places where the language could be a barrier and, in these situations, proper communication between the surgeon and the operating team should be ensured.

The operating room has additional broadcast equipment, there are additional people in the operating room for transmission support, there are prolonged anesthesia/surgical times (for explanations to the auditorium), all increasing the risk for infections [12]. All of these aspects should be discussed prior to the surgery, trying to minimize the risks. Moreover, the positioning of the additional broadcasting equipment should not impede the normal positioning of the patient or the surgical team.

Even if the surgeons are "at home", operating in front of many peers is a source of stress and, for sure, the situation is different from the normal conditions. That is why the proper surgeon selection is of paramount importance. He/she should not only be very competent but, equally important, eager to teach and cold-blooded, immune to live surgery, like a pilot in an air show. Theoretically, only their commitment to teaching (not their reputation, advancement in career, or their access to sponsorship) should be their reason to agree to operate in live surgery sessions. Only the proper surgeon would be able to stop the procedure when things are going bad and to avoid exposing the patient to further, unnecessary risks. The others, driven by other aims, would take all risks to prove themselves and to others that they can do it. There are surgeons who fulfill all requirements for the proper surgeon and others who do not [10]. Thus, selection (of the proper surgeon as well as the proper patients) is one of the most important tasks of the surgical live event local director.

During the procedure, the surgeon's attention should be split between the procedure itself and explaining the steps and the reasons for choosing a certain approach, answering the questions from the auditorium, etc. It can be compared to a driver speaking to a mobile phone while driving [4]. If the collaboration with the surgical team is not the best, the situation could become a nightmare for the surgeon. That is why there are recommendations to moderate the transmissions, having a member in the auditorium and one in the operating room [3].
They should reduce the pressure on the surgeon, especially in difficult steps of the procedures, considering that the majority of surgeons reported high levels of anxiety as they operated in live-surgery sessions, which were more important when they operated outside their home institution [9].

One of the less discussed aspects of the live-surgery sessions is related to the postoperative care and management of complications. Usually, due to the tight schedule, the guest surgeon does not follow the case more than 1-2 days. When complications occur, the host institution manages the case. Sometimes they can manage the case without problems, sometimes not. Many times, the guest surgeon does not even know about the complications, because he/she is not informed by the hosts [1]. If this happens, it is very difficult for the surgeon to decide how to manage the case, because he does not know the therapeutic possibilities in the host institution [1]. Beside the standard, common-sense approach of a certain complication, there are two extreme alternatives which could be chosen and should be avoided: either an aggressive one (e.g. a nephrectomy for a postoperative bleeding post-percutaneous nephrolithotomy) for "safety reasons" or an excessive passive one (prolonging the discomfort of the patient or, even worse, favoring more complex complications or even the death of the patient) for "not compromising the event, the surgeon reputation or the host institution". All these situations can lead to lawsuits and should be avoided by clearly defining the role and the procedures for postoperative care of the patient, and especially for the postoperative complications.

It is interesting to mention that in a survey of the American Association of Genitourinary Surgeons, $70.9 \%$ of the surgeons (of whom $93 \%$ performed live surgery at least once) considered live surgery sessions as ethically acceptable but only $30 \%$ considered that the actual form of live surgery sessions should be allowed to continue in the current form [4].

\section{The attendant's perspective - learn from the best and beyond}

If live surgical events are ethically acceptable for 93\% of attendants, why should they be modified (as $70 \%$ believed)? Due to safety reasons for the patients? It means that it is an unethical procedure. Should it be due to certain risks for the surgeons? 
It is, also, unethical to expose the surgeon to unnecessary risks/stress. Could the teaching value in an actual form which does not fulfill the attendee's education requirements have been the reason for answering the question in that manner? If so, it is unethical to organize such events as long as it does not match the attendees' needs. Thus, how can it be improved?

Certainly, surgical specialties need, besides theoretical knowledge, to see various procedures before doing them and, for sure, the future of every specialty is in the residents and fellows whom we educate. As there is tremendous development of new devices and techniques, the chance to learn them in a single center (the training center or the home institution) has become tougher. On the other hand, the number of urologists (surgeons) who need to improve their knowledge and skills is higher from one year to another. How to cope with this? What are the alternatives to live surgery sessions?

- By fellowships.

- By courses.

- By DVDs, video tapes or from internet.

Interestingly during the World Congress of Endourology in 2011, a survey among 256 urologists who previously attended live surgical sessions revealed that $66 \%$ considered that recorded procedures are far better than live surgery [1]. The small sample group and the increasing number of participants during other subsequent major live surgery sessions contradict the survey result. As we mentioned before, the number of attendees at the live surgical sessions of the EAU annual meeting are impressive. Why they are doing that? Probably "because they want to be familiar with tomorrow's technique and be ready for the real world" [10] and probably because they can interact with the surgeon, ask questions (even through the moderator), hear their peer opinions and ideas. So, in fact, the key element, beyond other factors, is the interaction, the feeling that they are involved in the procedure.

Undoubtedly there is also a need to see the whole procedures, with tips, tricks and pitfalls, with complications and their management, as these aspects are very important for a surgeon. However, it should be mentioned that usually, during a live session, at least two procedures are broadcasted, in order to "fill the time" and, when a complication occurs, the transmission is generally switched to another operation room, to allow the surgeon to solve the complication. Thus, the objective of seeing how to manage complications, especially the important ones, is rarely fulfilled. On the other hand, the case selection for such sessions is extremely careful, the surgery being generally easier than in real life. The repair ratio for live cardiac surgery is around $98 \%$, compared with a lower percentages in real life [10].

The broadcast session should be of high quality, without interruption, and all the participants should have the possibility to put questions (some of them will be answered by the moderator, while others will be transmitted to the surgeon). Another factor which ensures the success of a live surgery session is the number of participants. It seems that an audience of less than 100 people offers better conditions for interactivity and exchange of ideas. The larger the groups, the smaller the impact on the individual levels is noted [10].

\section{A glimpse toward the future}

Clearly, live surgery sessions play an important role in the surgeon's education although the opponents of live surgery consider that attending live surgery sessions by viewing recorded videos "...lacks drama and the potential for catastrophe that the audience may witness, reminiscent of the bloodlust of the games in ancient Rome!" [1]. Meanwhile, in quite a rude assumption from the pioneer of endourology, Vanermen, one of the most renowned cardiac surgeons, considers the video-recorded surgical procedures as a fake, idealized world, without a future [10]. Moreover, he considers that "Surgery during videotapes is embellished; videotapes invariably start with a bird's-eye view of the hospital and finish with the patient strolling along the beach. The images of the procedure that are not that nice are omitted. Thus, edited videotapes are an illusion" [10].

Then, a question is raised. Why would $70 \%$ of urologists from a survey among members of the American Association of Genitourinary Surgeons not agree to be patients in a live surgery session [9]? Does it means that ethical issues are still present? Or it is a prejudice?

However, it becomes clear that except fellowships, two major options remain for the surgical training: recorded surgical procedures and live surgery sessions. However, the tendency in the last years for video-recorded surgical procedures is to present unedited, pre-recorded surgical procedures 
which are presented by the surgeon from the auditorium. It can be an alternative to all of the inconveniences presented before.

Another alternative to a genuine live-surgery session is broadcasting the surgical procedure from the surgeon's host institution, reducing the problems presented before. Nowadays, there is no problem to broadcast in very good conditions a live surgery session offering all the advantages of the live surgery sessions and preventing most of the ethical, legal and clinical aspects discussed above.

\section{Conclusions}

Despite the numerous controversies and ethical aspects, it remains one of the favorite methods of learning or at least of first contact with a certain method. By recognizing, assuming, discussing and regulating all of the ethical, legal and clinical issues which are related to this kind of surgical education method, the method can be beneficial for patients, surgeons and trainees. Regulations and using it responsibly, not banning, seems to be the way which could offer the benefits and prevent unethical and dangerous aspects of the method.

\section{Conflict of interest}

The authors declare no conflict of interest.

\section{References}

1. Smith A. Urological live surgery - an anathema. BJU Int 2012; 110: 299-300.

2. Williams JB, Mathews R, D’Amico T. Reality surgery - a research ethics. Perspective on the live broadcast of surgical procedures. J Surg Educ 2011; 68: 58-61.

3. European Association of Urology - Live Surgery Endorsement Guide. Available online at: http://uroweb.org/wp-content/uploads/EAU-Live-Surgery-Endorsement-Guide.pdf

4. Philip-Watson J, Khan SAA, Hadjipavlou M, Rane A, Knoll T. Live surgery at conferences - clinical benefits and ethical dilemmas. Arab J Urol 2014; 12: 183-6.

5. Kimmelman J. The therapeutic misconception at 25: treatment, research, and confusion. Hastings Center Report 2007; 37: 36-42.

6. Horng S, Grady C. Misunderstanding in clinical research: distinguishing therapeutic misconception, therapeutic misestimation, and therapeutic optimism. IRB 2003; 25: 11-6.

7. American Academy of Ophtalmology - Advisory opinion of the code of ethics. Available online at: http://www.aao.org/ethics-detail/advisory-opinion-live-surgery

8. Schmit A, Lazaraki G, Hittelet A, et al. Complications of endoscopic retrograde cholangiopancreatography during live endoscopy workshop demonstrations. Endoscopy 2005; 37: 695-9.
9. Duty B, Okhunov Z, Friedlander J, et al. Live surgical demonstrations: an old, but increasingly controversial practice. Urology 2012; 79: 1185e7-11.

10. Vanermen $\mathrm{H}$. Live surgery should not be outlawed at national and regional cardiothoracic meetings. J Thorac Cardiovasc Surg 2010; 139: 822-5.

11. Mundy AR. Education by humiliation. In: Emergencies in Urology. Hohenfellner M, Santucci RA (eds.). Springer, Berlin 2007; 557.

12. Pryor F, Messmer PR. The effect of traffic patterns in the OR on surgical site infections. AORN J 1998; 68: 649-60.

Received: 29.12.2016, accepted: 25.02.2017. 